\title{
Identified Hadron Production from the RHIC Beam Energy Scan
}

\author{
Lokesh Kumar (for the STAR Collaboration) \\ Physics Department, Kent State University, Kent, OH-44242, USA. \\ E-mail: lokesh@rcf.rhic.bnl.gov
}

\begin{abstract}
A current focus at RHIC is the Beam Energy Scan to study the QCD phase diagram - temperature $(T)$ vs. baryon chemical potential $\left(\mu_{B}\right)$. The STAR experiment has collected data for $\mathrm{Au}+\mathrm{Au}$ collisions at $\sqrt{s_{N N}}=7.7 \mathrm{GeV}, 11.5 \mathrm{GeV}$, and $39 \mathrm{GeV}$ in the year 2010. We present midrapidity results on rapidity density, average transverse mass, and particle ratios for identified hadrons from the STAR experiment. Collision dynamics are studied in the framework of chemical and kinetic freeze-out conditions.
\end{abstract}

\section{Introduction}

The RHIC beam energy scan (BES) [1] started in the year 2010. Its main objectives are to search for the possible QCD phase boundary and to search for the possible QCD critical point in the QCD phase diagram. At low temperatures, the relevant degrees of freedom are expected to be hadronic but at high temperatures, the quarks and gluons are the relevant degrees of freedom of the system. In the QCD phase diagram, at $\mu_{B} \sim$ 0 , the transition from hadronic gas to quark gluon plasma (QGP) is expected to be a crossover [2]. At large $\mu_{B}$, it is expected to be a first order phase transition. The point where the first order phase transition line ends is called the QCD critical point.

From the spectra and ratios of the produced hadrons, $\left(T, \mu_{B}\right)$ space points on the QCD phase diagram can be obtained. Once the $\left(T, \mu_{B}\right)$ space points are obtained, one can study various signatures of the possible QCD phase boundary and QCD critical point. The data presented here are from $\mathrm{Au}+\mathrm{Au}$ collisions at $\sqrt{s_{N N}}=7.7,11.5$, and $39 \mathrm{GeV}$, for the midrapidity $(|y|<0.1)$ region using both the STAR Time Projection Chamber (TPC) and the Time Of Flight (TOF) detectors [3]. The errors shown in figures are statistical and systematic errors added in quadrature.

\section{Results and Discussions}

\subsection{Energy Dependence of Yields and Average Transverse Mass}

Figure 1 shows the energy dependence of yields per participating nucleon pair (top panels) and the quantity $\left\langle m_{T}\right\rangle-m$ (bottom panels) as a function of $\sqrt{s_{N N}}$ for $\pi^{ \pm}$ 

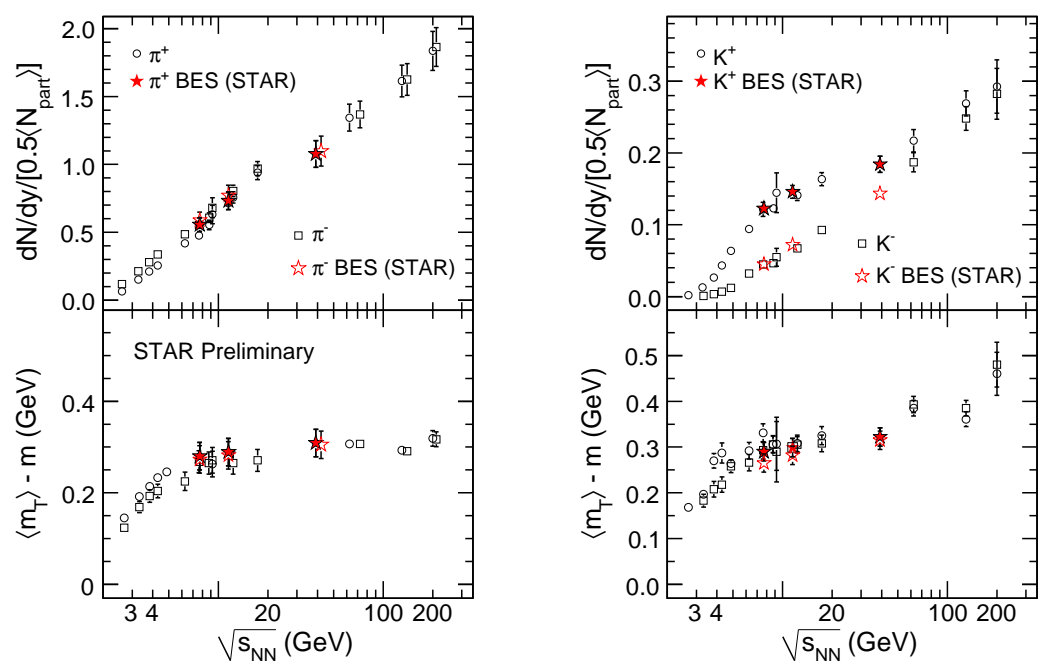

Figure 1. (color online) Left: $d N / d y$ divided by $\left\langle N_{\text {part }}\right\rangle / 2$ (top panel) and $\left\langle m_{T}\right\rangle-m$ (bottom panel) of $\pi^{ \pm}$, plotted as a function of $\sqrt{s_{N N}}$ for central collisions at midrapidity. BES results (star symbols) are compared with published data at RHIC 4, SPS [5], and AGS [6]. Right: Similar measurements for $K^{ \pm}$.

(left) and for $K^{ \pm}$(right). The pion yields for the BES data are corrected for weak decays feed-down and muon contamination using STAR HIJING+GEANT, as done for previous STAR results [4]. The BES results are consistent with the published energy dependence trend. The yields per participating nucleon pair increase with beam energy. The quantity $\left\langle m_{T}\right\rangle-m$, where $m_{T}=\sqrt{p_{T}^{2}+m^{2}}$ and $m$ is the hadron mass, increases with beam energy for lower energies, becomes almost constant for the region covered by the BES data and then tends to increase towards the top RHIC energies. This is an interesting observation in a scenario where the system is in a thermodynamic state. In that case, $\left\langle m_{T}\right\rangle-m$ can be related to the temperature of the system and $d N / d y\left(\propto \log \left(\sqrt{s_{N N}}\right)\right)$ may represent entropy. Then this observation could reflect the signature of a first order phase transition as proposed in ref. [7]. However, other interpretations of the observed $\left\langle m_{T}\right\rangle-m$ are possible [8].

\subsection{Particle Ratios}

Figure 2 (left panel) shows the correlation of the $K^{-} / K^{+}$ratio with the $\bar{p} / p$ ratio for new measurements from BES energies along with the published results [4]. This could give information on how the kaon production is related to the net-baryon density $(\bar{p} / p$ ratio). The STAR $p$ and $\bar{p}$ yields are not feed-down corrected. At lower energies, the kaon production is dominated by the associated production which results in more $K^{+}$ production compared to $K^{-}$. Also the $\bar{p} / p$ ratio is much less than unity, indicating that there is large baryon stopping at the lower energies. As we go towards higher energies, the pair production mechanism starts to dominate and the ratios tend to become closer 

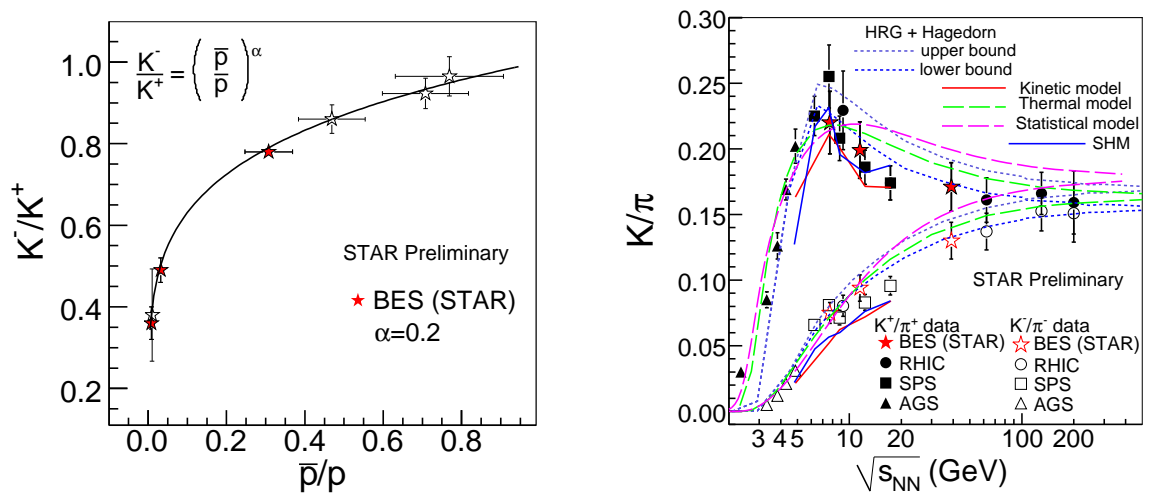

Figure 2. (color online) Left: Correlation of $K^{-} / K^{+}$ratio with $\bar{p} / p$ ratio for central collisions at midrapidity for different energies. Right: Energy dependence of $K^{ \pm} / \pi^{ \pm}$ ratio for central collisions at midrapidity. New results from BES data (star symbols) are compared to previously published results at RHIC [4], SPS [5], and AGS [], and are also compared with various theoretical model predictions [11.

to unity. The correlation between $K^{-} / K^{+}$(representing net-strange chemical potential, $\mu_{S}$ ) and $\bar{p} / p$ (representing net-baryon chemical potential, $\mu_{B}$ ) ratios seems to follow a power law behavior with $\alpha=0.2$ (represented by the curve). In a hadron gas, the relationship between $\mu_{S}$ and $\mu_{B}$ depends on the temperature. In a particular case of $T=190 \mathrm{MeV}$ and $\mu_{B}<500 \mathrm{MeV}$, these potentials follow the relation $\mu_{S}=(1 / 3) \mu_{B}[9]$.

Recent theoretical calculations [10] suggest that the maximum net-baryon density at freeze-out is attained at the lowest BES energy of $\sqrt{s_{N N}} \sim 7.7 \mathrm{GeV}$. The maximum netbaryon density could also be related to the peak observed in the energy dependence of $K^{+} / \pi^{+}$ratio at around $\sqrt{s_{N N}} \sim 7-8 \mathrm{GeV}$, as was observed by the NA49 experiment [5]. This is sometimes referred to as the "horn". The $K / \pi$ ratio could also suggest the strangeness enhancement in heavy-ion collisions with respect to the elementary collisions. Figure 2 (right panel) shows the energy dependence of $K^{ \pm} / \pi^{ \pm}$ratio for central collisions at midrapidity. The BES results are in good agreement with the trend of energy dependence established by the published measurements. The energy dependence of $K / \pi$ ratio seems to be best explained using HRG+Hagedorn model [11].

\subsection{Freeze-out Conditions}

The chemical and kinetic freeze-out conditions can be obtained by comparing ratios and spectra of the produced particles with the thermal equilibrium [4, 12] and blastwave (BW) [4, 13] model calculations. The main parameters extracted from these model comparisons are $T_{\mathrm{ch}}$ and $\mu_{B}$ for chemical freeze-out conditions, and $T_{\mathrm{kin}}$ and average flow velocity $\langle\beta\rangle$ for kinetic freeze-out conditions. Figure 3 shows the energy dependence of freeze-out parameters - $\mu_{B}$ (left panel), $T_{\text {ch }}$ and $T_{\text {kin }}$ (middle panel), and $\langle\beta\rangle$ (right panel), for central collisions. The $\mu_{B}$ decreases with the beam energy. This is expected since there are fewer net-baryons at midrapidity at higher energies because 

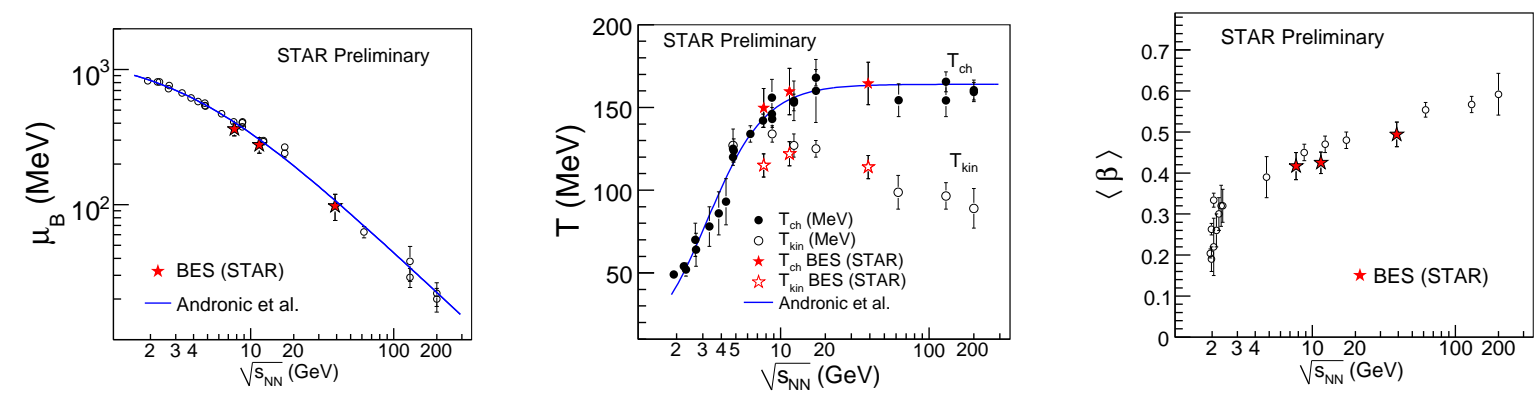

Figure 3. (color online) Energy dependence of baryonic chemical potential (left), temperature $\left(T_{\mathrm{ch}}, T_{\mathrm{kin}}\right)$ (middle), and average flow velocity (right). New results from BES data are shown with star symbols while other results are from ref. [4] and references therein. Lines represent the parametrization from ref. [14].

of less stopping of baryons at midrapidity at higher energies. The $T_{\mathrm{ch}}$ increases with energy and saturates at the higher energies while $T_{\text {kin }}$ decreases with beam energy after $\sqrt{s_{N N}} \sim 7.7 \mathrm{GeV}$. The $\langle\beta\rangle$ increases with beam energy.

In summary, bulk properties from the RHIC Beam Energy Scan are presented. The results are in good agreement with the energy dependence trend established by the published measurements. The yields of identified hadrons increase with beam energy. The quantity $\left\langle m_{T}\right\rangle-m$ is almost constant for the BES energies. The net-baryon density plays an important role at the lower energies as demonstrated by the energy dependence of $K / \pi$ ratio and correlation between $K^{-} / K^{+}$and $\bar{p} / p$ ratios. The new measurements from the BES program extend the $\mu_{B}$ range covered by RHIC from $20-400 \mathrm{MeV}$.

\section{References}

[1] B. I. Abelev et al. (STAR Collaboration), Phys. Rev. C 81, 024911 (2010); STAR Internal NoteSN0493, 2009; L. Kumar (STAR Collaboration), Nucl. Phys. A 830, 275C (2009).

[2] Y. Aoki et al., Nature 443, 675 (2006).

[3] M. Anderson et al., NIM A 499, 659 (2003); W. J. Llope et al., NIM B 241, 306 (2005).

[4] B. I. Abelev et al. (STAR Collaboration), Phys. Rev. C 79, 034909 (2009); ibid. 81, 024911 (2010).

[5] S. V. Afanasiev et al. (NA49 Collaboration), Phys. Rev. C 66, 054902 (2002); C. Alt et al. (NA49 Collaboration), Phys. Rev. C 77, 024903 (2008); ibid. 73, 044910 (2006);

[6] L. Ahle et al. (E866 Collaboration and E917 Collaboration), Phys. Lett. B 490, 53 (2000); ibid. 476, 1 (2000); J. L. Klay et al. (E895 Collaboration), Phys. Rev. Lett. 88, 102301 (2002).

[7] L. Van Hove, Phys. Lett. B 118, 138 (1982).

[8] B. Mohanty et al., Phys. Rev. C 68, 021901 (2008) and references therein.

[9] I. G. Bearden et al. (BRAHMS Collaboration), Phys. Rev. Lett. 90, 102301 (2003).

[10] J. Cleymans et al., Phys. Rev. C 74, 047901 (2006).

[11] S. Chatterjee et al. Phys. Rev. C 81, 044907 (2010); B. Tomasik et al. Eur. Phys. J. C 49, 115 (2007); J. Cleymans et al. Eur. Phys. J. A 29, 119 (2006); A. Andronic et al. Phys. Lett. B 673, 142 (2009); I. Kuznetsova et al. J. Phys. G 35, 044011 (2008).

[12] P. Braun-Munzinger et al., Phys. Lett. B 344, 43 (1995).

[13] E. Schnedermann et al., Phys. Rev. C 48, 2462 (1993).

[14] A. Andronic et al., Nucl. Phys. A 834, 237 (2010). 